\title{
Laparoscopic treatment of internal hernia following minimally invasive left hemicolectomy
}

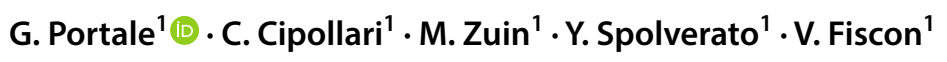

Received: 6 April 2020 / Accepted: 29 April 2020 / Published online: 22 May 2020

(c) Springer Nature Switzerland AG 2020

A 48-year-old female was referred to our unit for abdominal pain and bowel obstruction. She had undergone a minimally invasive left hemicolectomy for diverticular disease 9 months earlier. Colonoscopy, computed tomography (CT) colonography and a CT scan of the abdomen suggested internal hernia. The surgical management of the patient is shown in the attached video.

A laparoscopic approach with 4 ports was used: the camera in the umbilicus and 3 operative trocars in the right hypochondrium and iliac fossa and in the supra-pubic area. An initial distension of small bowel loops was found. However, this did not prevent the completion of a fully laparoscopic procedure. Several small bowel loops were found herniating through the mesenteric defect, which had been closed with glue in the previous operation, according to the surgical report. The herniated loops were reduced and found to be vital. The mesenteric defect was carefully closed with a double layer of 3-0 sutures. Care was taken not to injure the marginal arcade and compromise the vascularization of the transposed colon and its mesentery. The operation lasted $40 \mathrm{~min}$. The patient recovered well and was discharged on postoperative day 4 .

Internal hernia is a rare complication following laparoscopic colorectal surgery but should always be kept in mind [1-2]. A suspicion of internal hernia especially in symptomatic patients should prompt surgical revision to reduce the risk of finding an ischemic injury of the herniated loop(s). Early reoperation avoids resection of the ischemic bowel loop(s) and reduces morbidity and mortality risk for the patient.

\section{Compliance with ethical standard}

Conflict of interest The authors declare that they have no conflict of interest.

Ethical approval All procedures performed in studies involving human participants were in accordance with the ethical standards of the institutional research committee and with the 1964 Helsinki declaration and its later amendments or comparable ethical standards.

Informed consent Informed consent was obtained from all individual participants included in the study.

\section{References}

1. Toh JW, Lim R, Keshava A, Rickard MJ (2016) The risk of internal hernia or volvulus after laparoscopic colorectal surgery: a systematic review. Colorectal Dis 18:1133-1141

2. Portale G, Popescu O, Parotto M, Cavallin F (2019) Internal hernia after laparoscopic colorectal surgery: an under-reported potentially severe complication. a systematic review and meta-analysis. Surg Endosc 33:1066-1074

Publisher's Note Springer Nature remains neutral with regard to jurisdictional claims in published maps and institutional affiliations.
Electronic supplementary material The online version of this article (https://doi.org/10.1007/s10151-020-02233-6) contains supplementary material, which is available to authorized users.

G. Portale

portale@surgery.usc.edu

1 Department of Surgery, ULSS6 Euganea, Cittadella, Italy 